\title{
Lesson learned by COVID-19 outbreak: multilevel triage strategies in patients admitted to the emergency room in southern Italy
}

\author{
Pierpaolo Di Micco, ${ }^{1}$ Michele Imparato, ${ }^{1}$ Maria Teresa Iannuzzo, ${ }^{2}$ Andrea Fontanella ${ }^{1}$ \\ ${ }^{1}$ Department of Medicine, Ospedale Fatebenefratelli, Napoli; ${ }^{2}$ Health Management, Ospedale Fatebenefratelli, Napoli Italy
}

\begin{abstract}
After identifying a novel disease inducing a severe acute respiratory syndrome-related to coronavirus 2 (SARS-CoV-2) for epidemic pneumonia in China, the diffusion of infection is ongoing around the world, and Italy has been the second country in which an epidemic has been demonstrated. Clinical features of this infection may be summarized in viral pneumonia that SARS or acute respiratory distress syndrome (ARDS) may complicate. For this reason, this epidemic has been considerably more aggressive than the previous epidemic of SARS and Middle-East respiratory syndrome. Coronavirus disease 2019 (COVID-19) showed an easy diffusion from human to human and also showed to be more contagious than other viruses. So human contact should be avoided at the emergency room and for inpatients too. The Triage should be adapted to these new features in order to speed up procedures for the care of infected patients at high risk of morbidity and mortality for SARS and ARDS and for traditional access to the emergency room. Based on our experiences, this flow chart has been designed with a multi-level triage in which patients have been divided for admission to the emergency room into patients with fever/respiratory symptoms and patients without feverlrespiratory symptoms, to improve medical performances while treating COVID-19. This organized, multilevel triage permitted a good selection of patients admitted to the emergency room during the epidemic of COVID-19 in Southern Italy.
\end{abstract}

\section{Multilevel triage strategies in the Emergency Room}

After the recent Chinese epidemic of coronavirus disease 2019 (COVID-19) due to a novel coronavirus, ${ }^{1}$ Italy has been the second country in the world to have a similar epidemic that is currently ongoing. At present, inpatients for COVID-19 infection are more than $50 \%$ in Northern Italy, and are increasing up to $40 \%$ in Southern Italy. In this clinical setting, this new coronavirus is specific for respiratory tract infection and may also induce severe acute respiratory syndrome (SARS) with lung failure. ${ }^{1}$

Because of the relevant contagion of the virus, new effective strategies for selective triage in the Emer-

Correspondence: Pierpaolo Di Micco, Department of Medicine, Ospedale Fatebenefratelli, Napoli, Italy. E-mail: pdimicco@libero.it

Key words: COVID-19; coronavirus; SARS-CoV-2; emergency room; triage; coronaviridae family.

Received for publication: 10 December 2020

Revision received: 10 May 2021.

Accepted for publication: 10 May 2021.

This work is licensed under a Creative Commons Attribution NonCommercial 4.0 License (CC BY-NC 4.0).

${ }^{\circ}$ Copyright: the Author(s), 2021

Licensee PAGEPress, Italy

Italian Journal of Medicine 2021; 15:170-174

doi:10.4081/itjm.2021.1445 gency Room (ER) are needed to improve the selection of affected patients and to avoid the viral transmission, so escaping the diffusion of the virus epidemic. Compared to other epidemics of coronavirus that induced SARS and Middle-East respiratory syndrome (MERS), respectively in 2003 and 2012, COVID-19 seems to be able to induce clinical scenarios associated with increased morbidity and mortality according to data recently published on the Chinese population. ${ }^{2}$ COVID-19, in fact, is responsible for severe respiratory infections that may require admission to the hospital for interstitial pneumonia or SARS and then acute respiratory distress syndrome (ARDS). Furthermore, the clinical evolution of inpatients is longer than with other community-acquired pneumonia (CAP), so adding a further problem to the risk of depletion of medical resources for all hospitals.

So based on the experiences of the previous epidemic of SARS and MERS and based on the first epidemic of SARS-CoV-2 in China, in order to escape further diffusion of viral infection, a dedicated organization of triage, not only for ER but also for all other inpatients admitted for different reasons, has been suggested and used in many hospitals. ${ }^{3}$

In this way, patients admitted to ER that reeported symptoms not associated with fever, cough, respiratory dysfunction (i.e., chest pain, abdominal pain, headache, hemiparesis, aphasia) were addressed to regular triage. In contrast, patients with symptoms that could be associated with COVID-19 were admitted to a separate triage. This way, only the risk not to identify asymptomatic carriers of the virus was not evaluated. 
However, the triage system is based mainly on symptoms reported by patients associated with a thorough anamnesis and physical examination carried out by the triage members. Of course, this is a substantial limitation of the method, although it is common to every type of triage system.

On the other hand, patients with fever $>37.3^{\circ} \mathrm{C}$ and with $\mathrm{SpO}_{2}<93 \%$ should be early evaluated in the separate triage as also suggested by Zhang et al. ${ }^{3}$ as well as patients with sudden cough or dyspnea not previously experienced were evaluated in the dedicated triage. This viral infection, in fact, can be afebrile in the early stage but associated with cough and chill, so, also these symptoms should be taken into consideration for the separate triage. From a clinical point of view, physicians should detect early clinical signs as dyspnea, tachypnea, and hypoxemia that may suggest interstitial pneumonia. ${ }^{3}$ Useful laboratory tests for this separated triage that may speed up the diagnosis of COVID-19 are hemochrome with evidence of leukolymphopenia, increased CRP, fibrinogen, and LDH. So, patients with these clinical and laboratory characteristics should be addressed to early chest X-ray or chest CT scan to look for radiological signs of viral interstitial pneumonia. ${ }^{3}$ In addition, separated triage may benefit from a mobile or dedicated CT scan since the latter demonstrated an increased sensitivity than $\mathrm{X}$-ray in detecting specific radiological findings of COVID-19 as multiple bilateral infiltrations with a trend to become large ground-glass opacities. ${ }^{4}$

After this second step of triage, the differential diagnosis for the type of pneumonia can be better addressed. Samples made to detect viral pneumonia's pathogenesis should beanalyzed for seasonal flu subtypes or community-acquired pneumonia (CAP) or COVID-19.

While waiting for these microbiological tests, patients may benefit from treatments for hypoxia, fever, and dyspnea with additional oxygen, antipyretics, and antibiotics to prevent co-infection of Staphylococcus aureus or other bacteria. Patients positive for flu or CAP can be discharged from ER to specialist units of Pneumology or Infectious Disease or Internal Medicine, while patients positive for COVID-19 should be addressed to a separate specialist ward in which clinical observation of COVID-19 needs to be done. The clinical course of pneumonia induced by COVID-19 infection, in fact, may have complications such as SARS or ARDS. The treatment may be based on the administration of high flow oxygen alone or with CPAP support, ${ }^{5}$ glucocorticoids, ${ }^{3}$ moderate doses of chloroquine (e.g., $500 \mathrm{mg}$ twice daily), ${ }^{6}$ specific antivirals (e.g., lopinavir/ritonavir 200/50 mg twice daily or rendesmevir), ${ }^{7,8}$ antibiotics with high impact on the respiratory system as quinolones or cephalosporins and biologics (in particular tocilizumab or IgM en- riched immunoglobulins). ${ }^{9-11}$ However, for all indicated therapeutic support, being COVID-19 a newly identified disease, there is not a high level of evidencebased medicine or a specific guideline because, at present, there are few validated randomized clinical trials and $\backslash$ or controlled studies. So, frequently, different medical centers reported their clinical experience with each of these therapies. One of the most debated therapeutic support is the use of CPAP because of the risk of aerosolization that could facilitate viral diffusion, yet reported positive effects have been derived from previous outbreaks of SARS and MERS. ${ }^{5} \mathrm{Al}-$ though the risk of aerosolization is concrete, no study has demonstrated an effective increase of contagions.

Moreover, a multilevel triage strategy could be adopted not only for triage selection at the ER but also for the evaluation of inpatients with fever admitted to the hospital for other types of acute medical illness. In this clinical setting, in fact, data present in literature are lacking. So, a thorough clinical evaluation of patients that develop fever as a clinical complication of another acute disease that previously required admission to the hospital should be performed to evaluate differential diagnosis between COVID-19 and other causes of infection for inpatients. In addition, different daily clinical strategies and treatments may improve the clinical course of any type of infection and also accidental contamination of other inpatients.

So, we adopted an internal triage for inpatients that developed fever as a complication of other diseases that included laboratory tests, radiological imaging, and microbiological tests to identify fever associated with super-infection of COVID-19 in inpatients early (Figure 1).

According to available data supported by the Chinese epidemic of SARS-CoV-2, patients at risk of complications such as SARS or ARDS are elderly patients and patients with several comorbidities as well as immunocompromised patients for any reason (i.e., immunopathological diseases or recent immunotherapy or chemotherapy). For pregnant women, a consultant obstetric/gynecologicst should also consider evaluating pre-term delivery. ${ }^{11,12}$

For all other cases in which dyspnea is not associated with $\mathrm{SpO}_{2}<92-93 \%$ and fever is not higher than $37.2-37.3^{\circ} \mathrm{C}$ and clinical features do not suggest pneumonia, home care treatment or outpatients' clinic should be thoroughly suggested (Figure 2). Considering the significant number of patients involved in the epidemic that may ask for hospital admission, inappropriate admissions for less aggressive infections can be dangerous for patients who may have an overlapping co-infection by COVID-19 and the depletion of hospital resources.

Of course, further limitations to our procedural methods should be reported and based on social prob- 
lems besides technical problems. For example, in the demographic area of Naples, in Campania in Southern Italy, nearly one million citizens live, and in the hinterland of the city, there are about 2.5 million people with a very high density of population reaching the top ten cities in Europe. For this reason, each outbreak of viral or bacterial agents is really dangerous for the town and historically not infrequent also in the second half of XX century (e.g., epidemic of Vibrio cholerae).${ }^{13}$ So, each flu epidemic for the health care system is particularly hard to handle from a technical point of view, therefore, the triage organization has a relevant role.

For this reason, we planned a dedicated triage selection because we were not blind to the outbreak hypothesis. For the COVID-19 outbreak, in fact, based on data available from Wuhan and from Northern Italy that testified an increased rate of contagiousness of COVID$19,{ }^{14,15}$ with a really relevant rate of patients that would need intensive care unit supports, ${ }^{16}$ and that may quickly expand the viral transmission to all the community coming to the hospital, inducing depletion of medical services, all hospitals planned a different triage organization to counteract the viral diffusion between the different types of medical illness. We may affirm that this method contributed to the different mortality rates between Northern Italy and Southern Italy. ${ }^{16,17}$

Furthermore, a relevant practical limitation of this triage organization is represented by asymptomatic carriers of SARS-CoV-2 that are usually very numerous and dangerous for naïve people. For this reason, the admission to ER was allowed only to the patient

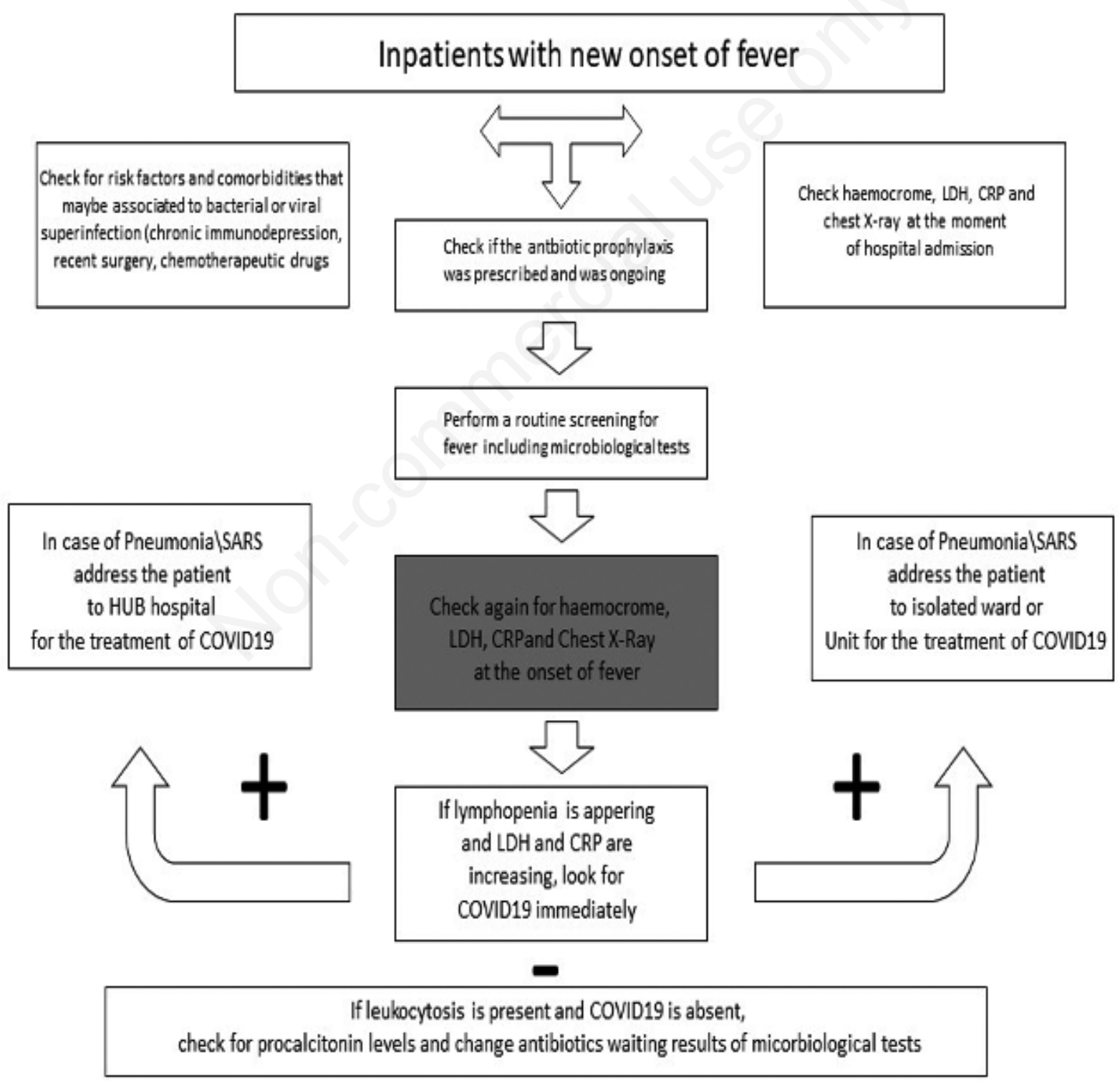

Figure 1. Internal triage and clinical strategies in patients with fever after admission in hospital for other acute illness. CAP, community-acquired pneumonia; CRP, C-reactive protein. 


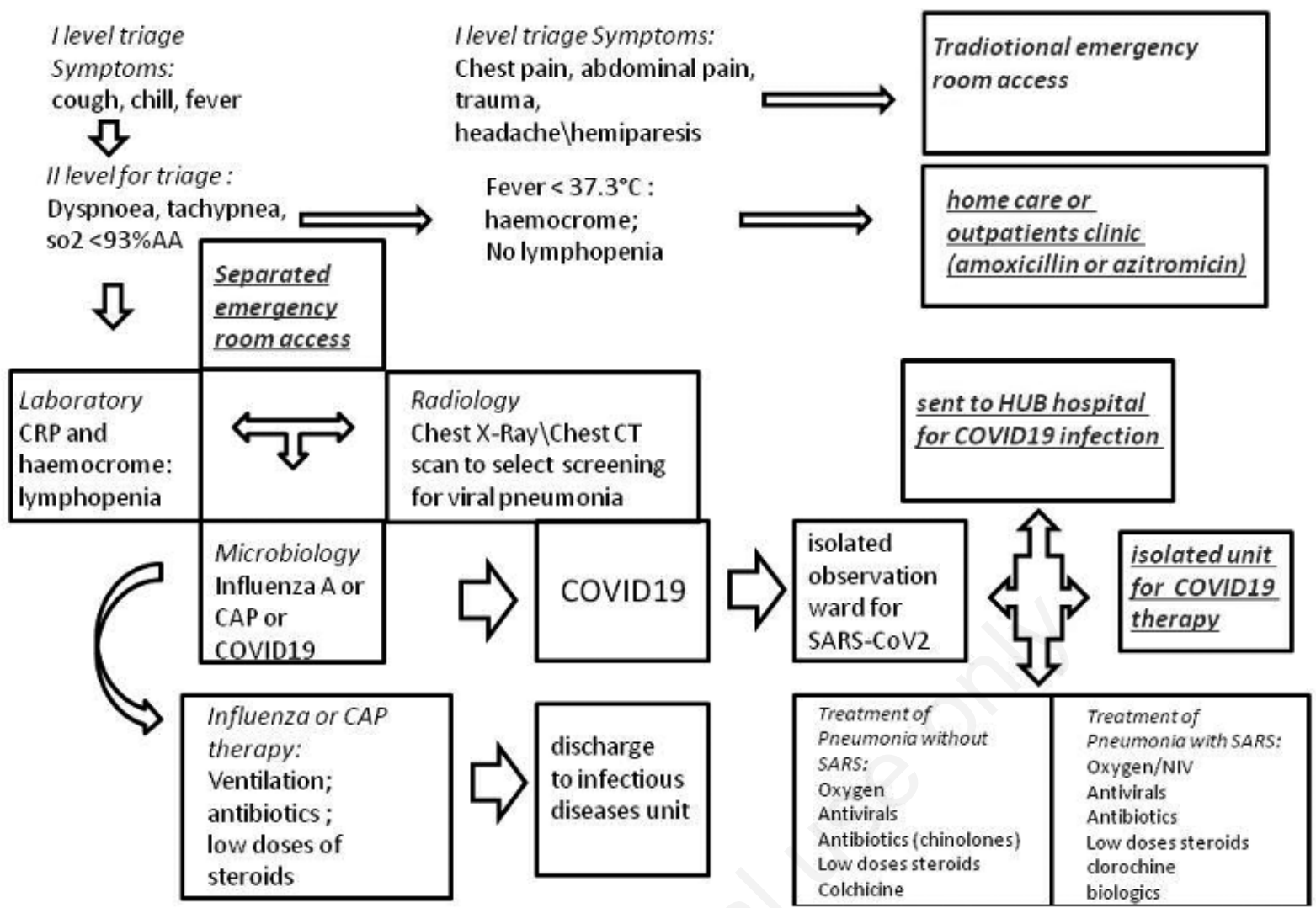

Figure 2. Clinical strategies for triage selection and destiny of patients with suspected novel coronavirus 2019 (COVID19) infections at Emergency Room. CRP, C-reactive protein.

with medical illness without any helper (family members or other people); furthermore, personal protective equipment were placed and worn by each patient in order to escape viral transmission to other patients, nurses, and physicians. ${ }^{18}$

So, many factors contributed to developing our clinical algorithm in Southern Italy during the epidemic period, particularly for patients admitted to the emergency room with suspected COVID-19 infection (Figure 2). During this time, the charge of patients to ER for fever clinics outweighed was very hard because of the contagion of COVID-19, associated with its increased morbidity and mortality and the risk of depletion for hospitals.

In conclusion, multilevel triage dividing patients with fever and patients without fever in separate wards within the ER was performed to have a double-check for hospital admission of patients and also to be adapted to the increased flow of patients with fever and suspected pneumonia by COVID-19 at high risk to develop life-threatening complication such as SARS that is associated with increased in-hospital mortality; of course, this protocol should be adapted to other conditions and epidemic specialists as the general public, public health professionals, clinicians, and other experts may adapt it for other requirements.

\section{References}

1. McCloskey B, Heymann DL. SARS to novel coronavirus: old lessons and new lessons. Epidemiol Infect 2020;148:e22.

2. Giwa A, Desai A. Novel coronavirus COVID-19: an overview for emergency clinicians. Emerg Med Pract 2020;22:1-21.

3. Veronese N, Demurtas J, Yang L, et al. Use of corticosteroids in coronavirus disease 2019 pneumonia: a systematic review of the literature. Front Med [Epub ahead of print].

4. Shi $\mathrm{H}$, Han X, Jiang N, et al. Radiological findings from 81 patients with COVID-19 pneumonia in Wuhan, China: a descriptive study. Lancet Infect Dis 2020;pii:S1473-3099(20)30086-4.

5. Hui DS. Severe acute respiratory syndrome (SARS): lessons learnt in Hong Kong. J Thorac Dis 2013;5:S122-6.

6. Rebeaud M, Zores F. Chloroquine use as antiviral treatment and the new SARS CoV 2. Front Med 2020 [Epub ahead of print].

7. Yhmj Lim J, Jeon S, Shin HY, et al. Case of the index patient who caused tertiary transmission of COVID-19 infection in Korea: the application of lopinavir/ritonavir for the treatment of COVID-19 infected pneumonia monitored by quantitative RT-PCR. J Korean Med Sci 2020;35:e79.

8. Hillaker E, Belfer JJ, Bondici A, et al. Delayed initiation 
of remdesivir in a COVID-19 positive patient. Pharmacotherapy 2020 [In press].

9. Shanmugaraj B, Siriwattananon K, Wangkanont K, Phoolcharoen W. Perspectives on monoclonal antibody therapy as potential therapeutic intervention for Coronavirus disease. Asian Pac J Allergy Immunol 2020;38:10-8.

10. Cellina M, Orsi M, Bombaci F, et al. Favorable changes of CT findings in a patient with COVID-19 pneumonia after treatment with tocilizumab. Diagn Interv Imaging 2020;pii:S2211-5684(20)30087-5.

11. Carannante N, Fiorentino G, Corcione A, et al. Administration of immunoglobulins in SARS-CoV-2-positive patient is associated with fast clinical and radiological healing: case report. Front Med (Lausanne) 2020; $7: 388$.

12. Rasmussen SA, Smulian JC, Lednicky JA, et al. Coronavirus disease 2019 (COVID-19) and pregnancy: what obstetricians need to know. Am J Obstet Gynecol 2020 [In press].

13. De Lorenzo F, Buogo A, Soscia M, Schisa C. Identification and treatment with a long-acting sulphonamide of 15 carriers of Vibrio cholerae El Tor serotype Ogawa, during the 1973 cholera epidemic in Naples. Chemotherapy 1977;23:345-55.

14. Zhang J, Zhou L, Yang Y, et al. Therapeutic and triage strategies for 2019 novel coronavirus disease in fever clinics. Lancet Respir Med 2020;8:e11-2.

15. Lippi G, Mattiuzzi C, Sanchis-Gomar F, Henry BM. Clinical and demographic characteristics of patients dying from COVID-19 in Italy versus China. J Med Virol 2020 [Epub ahead of print].

16. Grasselli G, Zangrillo A, Zanella A, et al.; COVID-19 Lombardy ICU Network. Baseline characteristics and outcomes of 1591 patients infected with SARS-CoV-2 admitted to ICUs of the Lombardy region, Italy. JAMA 2020 [Epub ahead of print].

17. Ministero della Salute. PCM-DPC dati forniti dal Ministero della Salute; aggiornammento del 16/04/2020. Available from: http://www.salute.gov.it/imgs/C_17_notizie_4505_0_file.pdf

18. Interim Guidance. Rational use of personal protective equipment for coronavirus disease 2019 (COVID-19); February 27, 2020. Geneva: World Health Organization. Available from: https://apps.who.int/iris/handle/10665/ 331215? show $=$ full 